\title{
Nephropathy in Diabetes
}

\author{
Authors \\ Ludwig Merker ${ }^{1}$, Bernd-Walter Bautsch², Thomas Ebert ${ }^{3,4}$, Martina Guthoff5 ${ }^{\text {, Berend Isermann }}{ }^{6}$
}

\author{
Affiliations \\ 1 MVZ DaVita Dormagen GmbH, Diabetes and Renal \\ Center, Dormagen, Germany \\ 2 Internal Medicine, Catholic Clinics Oberberg, \\ St. Josef Hospital, Engelskirchen, Germany \\ 3 University Hospital Leipzig, Department of \\ Endocrinology, Nephrology and Rheumatology, \\ Leipzig, Germany \\ 4 Karolinska Institutet, Department of Clinical Science, \\ Intervention and Technology, Division of Renal Medicine, \\ Stockholm, Schweden, Germany \\ 5 Medical Clinic IV, Diabetology, Endocrinology, \\ Nephrology, University Hospital Tübingen, Germany \\ 6 Institute for Laboratory Medicine, Clinical Chemistry and \\ Molecular Diagnostics, University Hospital Leipzig, \\ Germany
}

published online $\quad 4.1 .2021$

\author{
Bibliography \\ Exp Clin Endocrinol Diabetes 2021; 129: S60-S63 \\ DOI 10.1055/a-1284-6211 \\ ISSN $\quad 0947-7349$ \\ (C) 2021. Thieme. All rights reserved. \\ Georg Thieme Verlag KG, Rüdigerstraße 14, \\ 70469 Stuttgart, Germany
}

German Diabetes Association: Clinical Practice Guidelines

Diabetologie 2020; 15 (Suppl 1 ): S170-S174,

DOI:10.1055/a-1194-1458

\author{
Correspondence \\ Dr. Ludwig Merker \\ MVZ DaVita Dormagen GmbH \\ Diabetes and Renal Center \\ Elsa-Brändström-Str. 17 \\ 41540 Dormagen \\ Germany \\ ludwig.merker@davita.com
}

\section{Preliminary Note}

Patients with diabetes mellitus affected by kidney damage have an excessively increased cardiovascular risk. This requires an early diagnosis, a consistent and goal-oriented therapy of diabetes mellitus, arterial blood pressure and all concomitant diseases and an early, close and patient-centered cooperation between family doctor, diabetologist and nephrologist.

\section{Definition}

Nephropathy in diabetes is understood to represent all forms of kidney damage that may occur in temporal or causal relation to diabetes mellitus. In the course of the disease, there may be changes in the excretion of protein in the urine, a decrease in kidney function, recognizable by a reduction in the glomerular filtration rate, and the development and/or worsening of concomitant diseases associated with diabetes, such as arterial hypertension and lipid metabolism disorders.

The development of kidney damage in type 1 diabetes mellitus typically occurs in 5 stages [1]. In people with type 2 diabetes mellitus, the development of kidney damage may be different. Therefore, the extent of kidney damage is classified independently of the underlying disease according to KDIGO ( $\triangleright$ Fig. 1) by determining the estimated glomerular filtration rate (eGFR) and the extent of proteinuria/albuminuria [2].
Albuminuria is an important prognostic parameter for the progression of renal function impairment and is divided into 3 " $A$ " stages; the extent of renal function impairment is divided into 6 " $G$ " stages as an estimated glomerular filtration rate ( $>$ Fig. $\mathbf{1})$.

\section{Screening, Examination and Diagnosis}

At least once a year the eGFR and albuminuria should be determined in people with diabetes mellitus. The determination of albuminuria in morning urine is recommended. The ratio of albumin to creatinine (albumin-creatinine ratio, ACR), which allows a standardized and concentration-independent determination of the extent of albuminuria, is important. Before doing so, it is recommended to perform a urine multi-field strip test to exclude interfering factors. If the result is unclear or pathological, repeated determination at 3-month intervals is recommended for validation. Today, the determination of eGFR is usually performed according to the CKD-EPI formula or alternatively according to the MDRD formula.

Limitations of these estimation formulas include considerable excess weight or low weight, advanced age, but also rapidly changing kidney function.

\section{Differential diagnosis}

Not everyone affected by diabetes mellitus has kidney damage caused by diabetes in the presence of impaired kidney function 

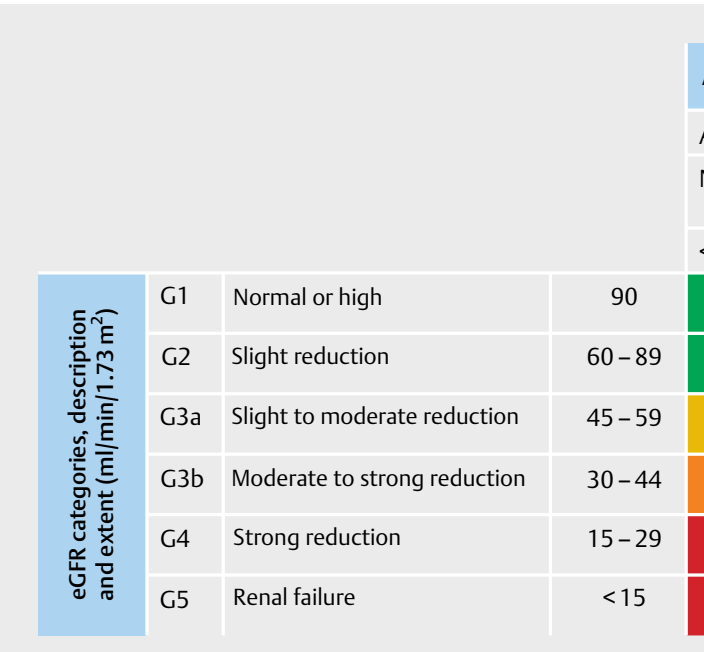

\section{Albuminuria stages, description and extent $(\mathrm{mg} / \mathrm{g})$}

\begin{tabular}{|l|l|l|}
\hline A1 & A2 & A3 \\
\hline Normal to mild increase & $\begin{array}{l}\text { Moderate increase, } \\
\text { microalbuminuria }\end{array}$ & $\begin{array}{l}\text { Severe increase, } \\
\text { macroalbuminuria }\end{array}$ \\
\hline
\end{tabular}

\begin{tabular}{l|l|l|}
$<30$ & $30-300$ & $>300$
\end{tabular}

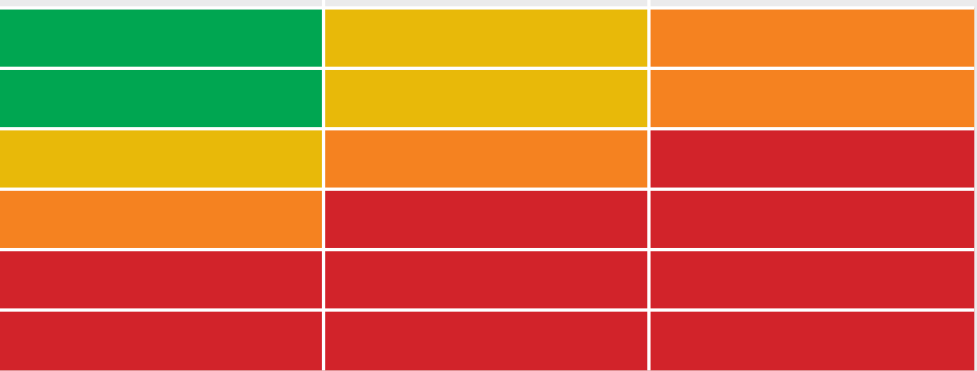

- Fig. 1 Stages of chronic renal failure according to KDIGO [2]. The risk of an unfavorable course is marked in color (green: low risk [if no markers of chronic kidney disease are present, no CKD]; yellow: moderately increased risk; orange: high risk; dark red: very high risk). CKD: chronic kidney disease; eGFR: estimated glomerular filtration rate.

and/or albuminuria. Close monitoring of the findings and the initiation of further diagnostic measures and/or an appointment with a nephrologist are necessary in the case of

- Patients with increased albuminuria/proteinuria:

- in case of suddenly-occurring high proteinuria

- with rapidly-increasing proteinuria

- in case of a rapid decrease of eGFR or increase of creatinine in serum

- for a pathological urine strip test, especially detection of erythrocytes and/or leucocytes

- for diabetes duration $\leq 5$ years for people with type 1 diabetes mellitus

- in case of conspicuous sonographic changes of the kidneys, asymmetrical kidney size, unilateral reduction in size, etc.

- Patients without albuminuria/proteinuria:

- they may have a hypertensive nephropathy; if arterial hypertension is present, further diagnostic measures may be necessary (see section on Blood Pressure).

\section{Supplementary diagnostics}

- Examination of the pulse status, especially in the area of the feet, determination of the ankle-brachial index, if necessary vascular Doppler examination

- Long-term blood pressure measurement

- ECG and/or stress test

- Fundoscopy

- Determination of lipid status, especially LDL cholesterol

\section{Prophylaxis and Delay of the Progression of Kidney Damage}

Lifestyle modification can have a beneficial effect on the development of kidney damage, for example, by

- Losing weight (decrease of proteinuria),

- Not smoking,

- If necessary, normalizing an increased protein intake.
General measures in everyday diagnostics and/or therapy:

- Restrictive use radiology

- Avoiding the administration of non-steroidal anti-inflammatory drugs

- Antibiotic therapy of symptomatic urinary tract infections

- Dose adjustment of medications to the reduced renal function and consideration of the risk of accumulation and interaction of medications in case of reduced renal function

Early and consistent therapeutic measures can positively influence the development and/or deterioration of kidney damage through

- Target value-oriented blood pressure therapy (see "Blood Pressure Therapy" section),

- Target value-oriented diabetes therapy (see "Therapy of Diabetes Mellitus" section),

- Target-oriented lipid therapy (see "Therapy of Dyslipidemia” section).

In close coordination with the nephrologist in charge, the therapy of renal disorders of the acid-base balance, possibly anemia, or the reduction of protein intake is carried out.

\section{Therapy}

\section{Blood pressure therapy}

Patients with type 1 and type 2 diabetes mellitus without manifest arterial hypertension

- In normotensive patients with type 1 and type 2 diabetes mellitus with persistent elevated albuminuria (stage A2 KDIGO), therapy with ACE inhibitors may be considered; this treatment should be performed for those with macroalbuminuria (stage A3 KDIGO). In the case of intolerance to ACE inhibitors, an AT1 blocker can be used as a substitute in both patient groups. 


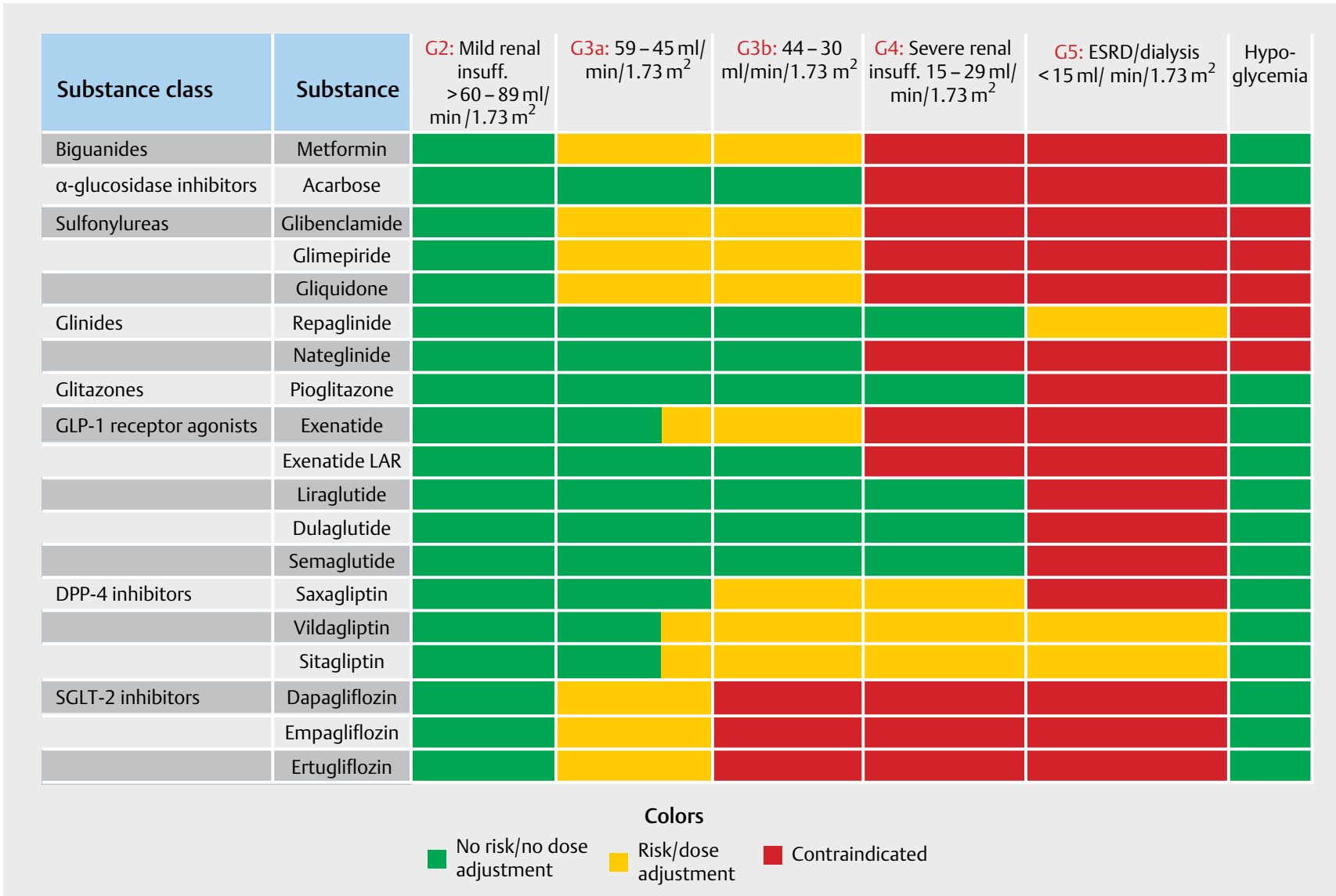

Fig. 2 Oral antidiabetic drugs that can be used in cases of impaired kidney function. Source: respective instructions for use for specialists, status $08 / 2020$.

Patients with type 1 and type 2 diabetes mellitus and arterial hypertension

- The ESC/ESH guidelines [3] recommend antihypertensive therapy for all patients with blood pressure $\geq 140 / 90 \mathrm{mmHg}$. Blood pressure should be reduced to target values $\leq 130 / 80 \mathrm{mmHg}$ in patients with hypertension and diabetes. In patients with chronic renal insufficiency a systolic target blood pressure of $130-139 \mathrm{mmHg}$ is recommended. However, the target blood pressure can be adjusted individually depending on the extent of proteinuria, concomitant diseases and therapeutic safety.

- For primary use in drug therapy:

ACE inhibitors - AT1 blockers in case of intolerance - in combination with calcium antagonists as well as other substances if necessary. Beta-blockers and diuretics are recommended as additional combination medications for the prevention of coronary complications and for blood pressure control; data on the possible deterioration of glycemic control is mixed.

The combined therapy of ACE inhibitors and AT1 blockers and, if necessary, aldosterone antagonists is not recommended because of the dangerous hyperkalemia that can occur.

\section{Therapy of diabetes mellitus}

- To prevent kidney damage, an HbA1c value between $6.5 \%$ $(48 \mathrm{mmol} / \mathrm{mol})$ and $7.5 \%(58 \mathrm{mmol} / \mathrm{mol})$ is recommended. The upper target range between 7.0 and $7.5 \%$ (53-58 mmol/ $\mathrm{mol}$ ) is recommended for patients with macroangiopathy and/or hypoglycemia perception disorder.

- To prevent the progression of diabetic kidney damage, an $\mathrm{HbA} 1 \mathrm{c}$ value of $\leq 7 \%(\leq 53 \mathrm{mmol} / \mathrm{mol})$ is recommended.

For patients with type 2 diabetes mellitus and a clinically-predominant chronic renal insufficiency, a sodium/glucose co-transporter-2 (SGLT-2) inhibitor therapy is recommended in addition to lifestyle and metformin therapy in the absence of contraindications. If this is not possible, glucagon-like peptide-1 (GLP-1) receptor agonists with proven cardiovascular benefit should be used $[4,5]$.

From stage G3b/G4 KDIGO on, the HbA1c value is no longer a reliable parameter for the quality of metabolic control, as it can be falsely low, especially in the presence and/or treatment of renal anemia.

For people with type 2 diabetes mellitus, $\triangleright$ Fig. 2 shows an overview of the selection and possible dose adjustment of some antidiabetic drugs in patients with impaired kidney function. It should be noted that people with diabetes and impaired renal function have a massively increased risk of hypoglycemia; this is due to a 
slower breakdown of insulin (especially exogenously-supplied insulin), reduced gluconeogenesis of the kidney, and prolonged renal elimination of oral antidiabetics with consecutively extended halflife. Therefore, renal function should be checked every 3/6/12 months depending on the stage of the disease, and the dose of antidiabetic drugs used should be adjusted in accordance with the concomitant medication.

\section{Therapy of dyslipidemia}

According to the ESC/EAS guidelines [6], the cardiovascular risk of patients in eGFR stages G3-G5 should be classified as high or very high. The presence of diabetes also increases the cardiovascular risk. For patients with chronic renal insufficiency not requiring dialysis, a statin or statin/zetimib combination is indicated. For dialysis patients without atherosclerotic cardiovascular disease, statin therapy should not be started again. However, if lipid-lowering therapy was in place before the start of dialysis, it can be continued.

\section{Inhibition of progression}

The risk of progression of renal failure is significantly determined by the extent of albuminuria and the limitation of renal function. The diabetes-related changes in the glomeruli are at least partially reversible, in principle, with optimally-controlled parameters for blood pressure, especially when the renin-angiotensin-aldosterone system (RAAS) is blocked, and blood glucose. In addition, blockage of the RAAS has been shown to be cardioprotective. It is possible that newer substances, which specifically influence the aldosterone effect, are similarly effective. Antidiabetic therapy with specific incretin mimetics [7] and SGLT-2 inhibitors [8] was shown to be effective in large cardiovascular endpoint studies (iraglutide: Leader, Dulaglutide: Rewind; Empagliflozin: EmpaReg-Outcome, Canagliflozin: Canvas, Dapagliflozin: Declare-TIMI-58) show a reduction in cardiovascular mortality and a consistent positive effect on albuminuria and eGFR loss over the study period, thus demonstrating a nephroprotective effect.

The Credence and Dapa-CKD studies, which were specifically designed to reduce renal endpoints, impressively demonstrated the nephroprotective effect of canagliflozin and dapagliflozin $[9,10]$. In almost all studies and meta-analyses, the nephroprotective effects were also shown in patients with impaired renal function. Therefore, it will be necessary to discuss whether the restriction on the use of SGLT-2 inhibitors should be removed in patients with impaired renal function. Further clinical studies on nephroprotection of SGLT-2 inhibitors are ongoing or planned.

\section{Follow-up}

Depending on the stage of nephropathy, the following parameters should be checked 2-4 times a year

- Determination of the eGFR

- Determination of albuminuria

- Blood pressure therapy including self-monitoring and possibly long-term blood pressure measurement

- Determination of the HbA1c value

- Determination of lipids (especially LDL cholesterol, possibly triglycerides)
In the case of renal insufficiency from stage G3a KDIGO (see - Fig. 1), patients with diabetes mellitus are recommended to consult a nephrologist. As renal function decreases with age for physiological reasons, the appointment with the nephrologist for diabetics older than 75 years should be made at the latest at stage G3b KDIGO. Any more severe kidney function impairment or findings indicating non-diabetic kidney disease require an immediate appointment with a nephrologist.

\section{Conflict of interest}

LM has received fees and/or reimbursements from the following companies/institutions in the last 3 years: AstraZeneca, Bayer, Boehringer Ingelheim, Bristol-Myers Squibb, Eli Lilly Pharma, Kassenärztliche Bundesvereinigung, Merck Inc., MSD Sharp \& Dohme, Novo Nordisk, Praxisnetz Dormagen, Servier. He declares that he sees no conflict of interest with the article he has created. TE has received fees/cost reimbursements from Lilly, Novo Nordisk and Sanofi over the last 3 years. Within the scope of a consulting activity he received a fee from the companies Sanofi and Boehringer Ingelheim Pharma. He is also supported by a Novo Nordisk Postdoctoral Fellowship, which is carried out in cooperation with the Karolinska Institutet, and by a mentorship program of the European Foundation for the Study of Diabetes (EFSD), which is supported by an Educational grant from AstraZeneca. MG received fees/ cost reimbursements/study grants from the following companies: Alexion, Astellas, Baxter, Bayer, Chiesi, AstraZeneca. B-WB and BI declare that they have no potential conflicts of interest.

\section{Literature}

[1] Mogensen CE. Introduction: Diabetes mellitus and the kidney. Kidney Int 1982; 21: 673-675

[2] KDIGO. KDIGO 2012 Clinical Practice Guideline for the Evaluation and Management of Chronic Kidney Disease. Kidney Int Suppl 2013; 3: 1-150

[3] Williams B, Mancia G, Spiering W et al. 2018 ESC/ESH Guidelines for the management of arterial hypertension. Eur Heart J 2018; 39: 3021-3104

[4] Buse JB, Wexler DJ, Tsapas A et al. 2019 update to: Management of hyperglycaemia in type 2 diabetes, 2018. A consensus report by the American Diabetes Association (ADA) and the European Association for the Study of Diabetes (EASD). Diabetologia 2020; 63: 221-228

[5] Landgrave R, Aberle J, Birkenfeld AL et al. Therapy of type 2 diabetes. Diabetol metabolism 2019; 14: S167-S187

[6] Mach F, Baigent C, Catapano AL et al. 2019 ESC/EAS Guidelines for the management of dyslipidaemias: lipid modification to reduce cardiovascular riskThe Task Force for the management of dyslipidaemias of the European Society of Cardiology (ESC) and European Atherosclerosis Society (EAS). Eur Heart J 2020; 41: 111-188

[7] Kristensen SL, Rørth R, Jhund PS et al. Cardiovascular, mortality, and kidney outcomes with GLP-1 receptor agonists in patients with type 2 diabetes: a systematic review and meta-analysis of cardiovascular outcome trials. Lancet Diabetes Endocrinol 2019; 7: 776-785

[8] Zelniker TA, Wiviott SD, Raz I et al. SGLT2 inhibitors for primary and secondary prevention of cardiovascular and renal outcomes in type 2 diabetes: a systematic review and meta-analysis of cardiovascular outcome trials. The Lancet 2019; 393: 31-39

[9] Perkovic V, Jardine M], Neal B et al. Canagliflozin and Renal Outcomes in Type 2 Diabetes and Nephropathy. N Engl J Med 2019; 380: 2295-2306

[10] Heerspink HJL, Stefánsson BV, Correa-Rotter R et al. Dapagliflozin in Patients with Chronic Kidney Disease. N Engl J Med. 2020; doi:10.1056/ NEJMoa2024816. Online ahead of print 